John A. Bal1

Harvard-Smithsonian Center for Astrophysics

60 Garden Street

Cambridge, Massachusetts 02138 USA

ABSTRACT. Why are we unaware of extraterrestrial intelligence?

The most interesting scientific problem of our age involves the question of the existence of extraterrestrial intelligence (ETI). But this is not to say that we here today are approaching the problem optimally or even rationally. I believe that mankind will be able to take over our galaxy, using unmanned probes, in a fairly short time--say a few hundred-thousand years--unless somebody else already has. Our current world view seems to predict the existence of other civilizations, much older than ours, who might have taken over the galaxy eons ago. Interacting with us should be easy for them. Why haven't they?

0lbers, in 1825 or so, was certainly not the first person to notice that the sky is dark at night; but he may have been the first to demand that this commonplace fact must accord with cosmological theory, else that theory is wrong. As olbers pointed out, the then-popular cosmology predicted that the sky should be as bright as the surface of a typical star. Such conflicts between theory, especially well-established theory, and facts, no matter how commonplace, yield paradigm shifts and scientific progress.

Another commonplace fact is that we are unaware of the existence of ETI, and this fact must accord with our world view, else that world view is, at least in part, wrong. My case is not as strong as 0lbers's. Indeed mine is more a prediction that this fact will come into conflict with our world view as it develops over the next few years, in particular when we establish that there are lots of extrasolar planets and that life forms and evolves wherever and whenever it can.

Where is everybody? With our present ignorance, we can only classify the possible answers to this question and estimate the likelihood of the correct answer falling in each category. Some of the categories, alas, are outside the realm of science. I want to discuss ten categories of possible answers to the question: Where is everybody?

Category (1): There are no other civilizations (OCs). Earth is either unique--the only biosystem--or mankind is first in level of development. Michael Hart and Frank Tipler, for example, argue, in essence, that absence of evidence $\frac{\text { is evidence of absence. One or }}{483}$ 


\section{Spectrum of Possibilities}

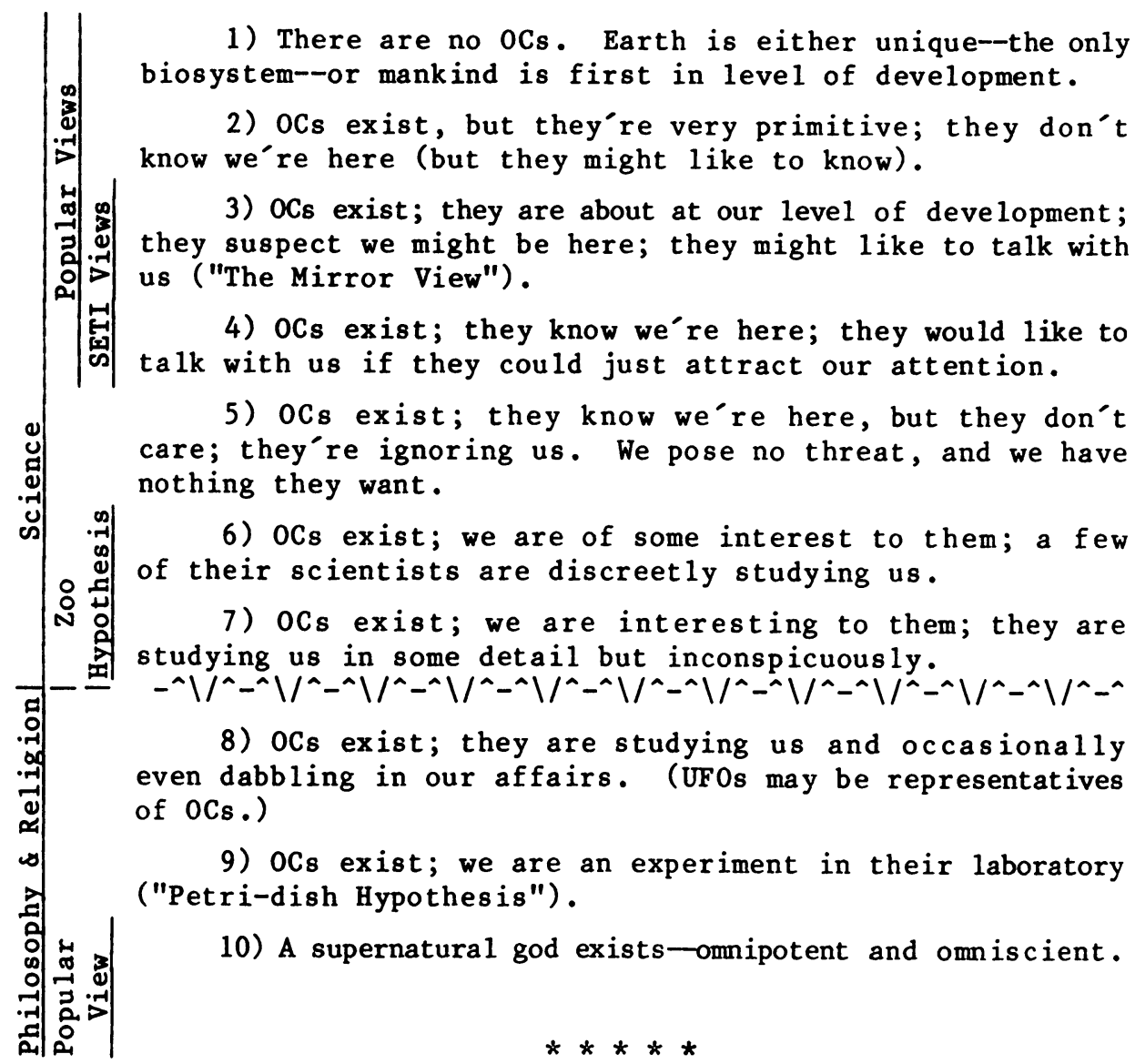

another of our starting premises might be wrong. I regard this answer as unlikely because we seem to lack any special situation that would make us singular or outstanding. I feel, nevertheless, considerable rapport with Hart and Tipler, although I think they' 11 eventually be shown to be wrong. They have asked the right question, and that's very important.

Category (2): Other civilizations exist, but they're very primitive. They don't know that we're here, but they might like to know. Their development has somehow been arrested at a level more primitive than ours. Category (3): Advanced civilizations exist at about our level of development. They suspect that we might be here, and they might like to talk with us. This hypothesis is called the "Mirror View" because we seem to see that ETI is just like us. The mirror, I suggest, is not very far from the end of our noses, and it's blocking the view of other things. Category (4): Advanced civilizations exist and they know we're here. They might like to talk with us if they could just attract our attention. 
Categories (2) through (4) are the popular views at least among my colleagues in radio astronomy. Categories (3) and (4) are the "official" views in the 1977 NASA report entitled The Search for Extraterrestrial Intelligence. I think these answers are wrong primarily because they vastly underestimate the abilities of ETI. Surely they know we're here, for the sun, the solar system, Earth, and mankind are all conspicuous; there's no way to hide. We'll certainly start studying and cataloging biosystems in the galaxy as soon as we"re able; why should ETI do less? Number (2) or (3) might be right if all civilizations, when they reach about our level of development, inevitably destroy themselves.

I suggest also that if advanced civilizations had chosen to announce their presence to us, we would be aware. Since we're not, I presume they have not. Look for ETI on Earthlike planets? More likely an Earthlike planet is to ETI what an empty eggshell is to a bird.

Category (5) (of answers to the question: Where is everybody?): They know we're here, but they don't care; they're ignoring us. We pose no threat, and we have nothing they want. This is a likely but a very unpopular answer, for it seems to downgrade mankind's importance, and we do like to feel important. We think we're pretty interesting; why shouldn't ETI think so too?

Categories (6) and (7) (of answers to the question: Where is everybody?): ETI may be discreetly and inconspicuously watching us but not dabbling. This is the zoo hypothesis, and for obvious reasons (Ba11, 1973), it's appealing to me. Kuiper and Morris (1977) point out that "Complete contact with a superior civilization (in which their store of knowledge is made available to us) would abort [our] further development ..." The joy of intellectual discovery would no longer be available; almost everything is already known to advanced civilizations, they need only teach us. Being a perpetual pupil is not to my liking; $I^{\prime} d$ rather do research, by which I mean discover something new--unknown to anyone else. Absorbing knowledge is less satisfying than creating knowledge.

According to this view, avoiding overt interaction is probably desirable both for ETI and for us. Mankind and ETI might be said to have a symbiotic relationship. Some of the alternatives involve our cultural extermination.

Although cultural interaction often destroys the lesser culture, there's actually little danger of this happening to us because real cultural interaction implies cultures relatively similar in developmental age; they must be able to exchange memes. In our whole galaxy there may be no civilization similar enough to ours for such destructive interaction to occur. The competitive exclusion principle in biology predicts that species can destroy one another when they directly compete for the same resources. Interaction with the most advanced civilizations would be on their terms, of course, but might be much less dramatic and destructive. Such an interaction, indeed, may already be taking place without our understanding.

Benign may not be quite the right word; but if you believe that there is someone out there with the ability to squash us like a bug, and he has not--we're still alive and functioning--this implies either that 
we're more valuable to him alive than squashed, or at least that we're not very detrimental to whatever he's doing.

We must be wary of slipping off the craggy cliff edge that forms the boundary between the solid ground of science and the mists and vapors of philosophy and religion. Categories (8) through (10) are outside the realm of science. If advanced civilizations are dabbling in our affairs, then the results of our experiments and observations and the conclusions based on them are suspect, and the foundations of the edifice of our science crumble. But saying that a hypothes is is not scientific is very different from saying that it's wrong.

Various combinations of these categories are possible. We might have, for example, (10) together with any of the others; and we might have one civilization, say in category (7), another in category ( 2 ), and so on. Some such combination, indeed, seems to me to be the most likely answer.

Asking the right question is sometimes remarkably important, especially in suggesting experiments and observations to be performed, and asking the wrong question can sometimes lead one far astray. I feel that this is just what is happening to those who are asking: How can we become aware of ETI? A better question, in my view, is: Why are we unaware of ETI?

\section{REFERENCES .}

Ba11, J.A., "The Zoo Hypothesis," Icarus 19:347 (1973).

Kuiper, T.B.H., and M. Morris, "Searching for Extraterrestrial Civilizations," Science 196:616 (1977).

Morrison, P., J. Billingham, and J. Wolfe, eds., The Search for Extraterrestrial Intelligence: SETI, National Aeronautics and Space Administration, NASA SP-419, Washington (1977).

$$
* * * * *
$$

